\title{
CORRELATIONS OF MECHANICAL STRESSES AND MAGNETICAL BEHAVIOUR OF ION IMPLANTED MAGNETO-OPTICAL LAYERS
}

\author{
F. HUBENTHAL AND K. RÖLL \\ University of Kassel, Heinrich-Plett Str. 40, 34132 Kassel, Germany
}

\begin{abstract}
Magneto-optical materials such as $\mathrm{TbFeCo}$ are used for data storage in a wide range. Especially the perpendicular magnetic anisotropy is necessary for high density recording. But the origin of the perpendicular magnetic anisotropy in these magneto-optical layers is not exactly known. In our experiments magneto-optical layers were implanted with heavy ions, with the aim to change the magnetic behaviour of these layers.. After the implantation there is a dramatical change in the magnetical and mechanical behaviour, too.
\end{abstract}

PACS numbers: $61.72 . \mathrm{WW}_{\mathrm{w}}$

\section{The samples}

We present results for thin film systems with one or two magneto-optical (MO) layers ("single" or "double"), which are prepared by rf-sputtering. The single layer system has one MO-layer of $53 \mathrm{~nm} \mathrm{TbFeCo,} \mathrm{which} \mathrm{is} \mathrm{sputtered} \mathrm{by} \mathrm{an}$ argon flux $\left(\Phi_{\mathrm{Ar}}\right)$ of $60 \mathrm{sccm}$ and a cover layer of reactively sputtered $30 \mathrm{~nm}$ SiN $\left(\Phi_{\mathrm{N}_{2}}=18.8 \mathrm{sccm}\right.$ and $\left.\Phi_{\mathrm{Ar}}=45 \mathrm{sccm}\right)$. The resulting magnetic moment is dominated by the iron sublattice magnetisation and a normal perpendicular magnetic anisotropy (PMA) hysteresis loop is visible in the Kerr magnetometer. In the double layer system the SiN and the first MO-layer are identical with the single-layer system but there is an additional $270 \mathrm{~nm}$ MO-layer under the first MO-layer. This bottom layer is sputtered by $\Phi_{\mathrm{Ar}}=80 \mathrm{sccm}$ and the composition of the layer changes. The resulting magnetic moment is now dominated by the Tb-sublattice magnetisation and one observes an inverted PMA hysteresis loop in the Kerr magnetometer. Furthermore, the first layer couples to the bottom one and the sublattice magnetisations are then orientated parallel to the bottom layer. In this case one observes an inverted hysteresis loop in the first MO-layer too, although it is iron dominated.

As substrates, glass plates with a thickness of $0.2 \mathrm{~mm}$ and a diameter of $12 \mathrm{~mm}$ are used for the magnetic measurements. For the stress measurements we use float-glass substrates D 263 made from Borsilikatglas by Deutsche Spezialglas $A G$, which are characterized by isotropic mechanical properties. 


\section{Instruments}

For the magnetic measurements we use a common polar Kerr magnetometer which is sensitive to the $\mathrm{Fe}$-sublattice magnetisation. It allows both measurements from the top and from the bottom side.

- The stresses are determined by a two-dimensional bending plate method, where the shape of the substrate is measured before and after deposition or implantation respectively, by a phase sensitive three-step Michelson interferometer, described elsewhere $[1,2]$.

The implantation was made at the ion implanter at the Gesellschaft für Schwerionenforschung in Darmstadt (Germany). The chosen ions, energies, and average deposition depths are listed in the following table. The deposition depths are calculated with the TRIM [3] program.

\section{TABLE}

Examples of energies and average deposition depths for several ions.

\begin{tabular}{l|c|c|c}
\hline \hline Layer system & Ions & Energy in keV & Average deposition depth \\
\hline single & $\mathrm{Tb} / \mathrm{Co} / \mathrm{Cu}$ & $153 / 100 / 93$ & $45 \mathrm{~nm}$ \\
double & $\mathrm{Cu}$ & 60 & $30 \mathrm{~nm}$
\end{tabular}

\section{Results}

An important and surprising experimental result was that there is no dependence on the kind of ions. There are no different effects in using rare earth $(\mathrm{Tb})$, transition metal $(\mathrm{Co})$, or non-magnetic materials $(\mathrm{Cu})$. Because of this, all of our results are discussed for copper ions only.

\subsection{Single layer system}

In this system the ions are implanted in an average depth of $45 \mathrm{~nm}$ (i.e. $15 \mathrm{~nm}$ in the MO-layer). Figure 1 shows the dramatical change of the magnetic anisotropy in dependence on the copper fluence. The PMA switches at last to completely in-plane magnetic anisotropy at a fluence of $1 \times 10^{15}$ copper ions per $\mathrm{cm}^{2}$. This effect is measured from both sides of the layers, which means that the complete MO-layer has changed to an in-plane anisotropy and not only the penetrated first nanometers. In the range between the both states perpendicular and in-plane anisotropy exist at the same time. In the stress measurements there is an enormous change as well, because the compressive stresses from the sputtering process disappear in the same way as the PMA. In Fig. 2 the change of the stresses in dependence of the fluence and for comparison the average stresses from the sputtering process are shown.

The internal stresses of $-0.6 \mathrm{GPa}$ disappear to zero exactly at the same fluence $\left(1 \times 10^{15}\right.$ ions $\left./ \mathrm{cm}^{2}\right)$ where the magnetic anisotropy changes completely to in-plane one. We find also transition states in the range from $1 \times 10^{13}$ up to $5 \times 10^{14}$ copper ions per $\mathrm{cm}^{2}$ where the stresses were partially relaxed. Furthermore, 


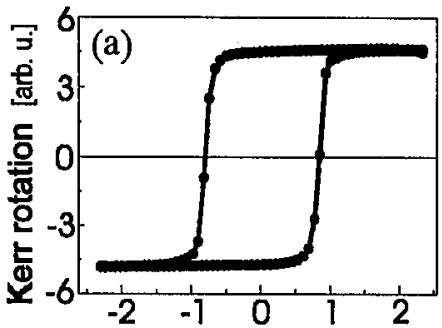

$B$ [T]

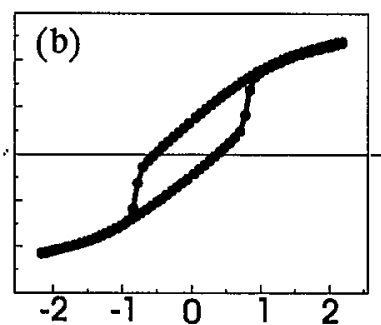

$B$ [ T ]

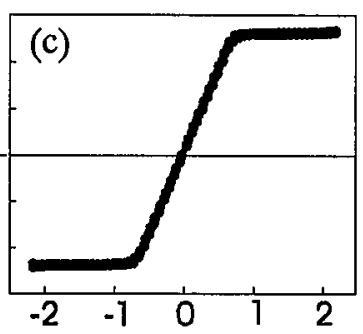

$\mathrm{B}[\mathrm{T}]$

Fig. 1. Change of the PMA in dependence on the applied fluence in the single layer. (a) Unimplanted sample, (b) $F=5 \times 10^{13}$ ions $/ \mathrm{cm}^{2}$, (c) $F=1 \times 10^{15} \mathrm{ions} / \mathrm{cm}^{2}$.

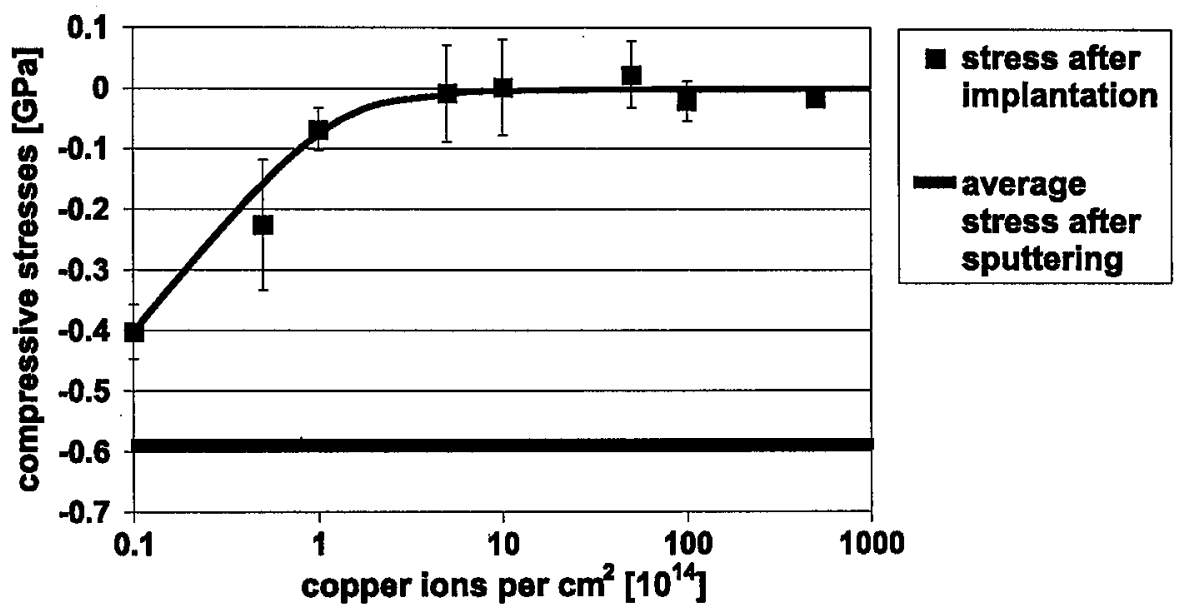

Fig. 2. Change of the internal stresses in dependence on the applied fluence.

no additional changes in both mechanical and magnetic behaviour were found at higher fluences up to $1 \times 10^{17}$ copper ions per $\mathrm{cm}^{2}$.

\subsection{Double layer system}

In this case the copper ions had an energy only of $60 \mathrm{keV}$ and therefore an average deposition depth of $30 \mathrm{~nm}$, which means that they stop exactly in the interface between the SiN cover layer and the first MO-layer. Again there is . a stress relaxation but evidently smaller as in the former experiments (Fig. 3a). One can see a saturation effect in the stress relaxation at $-0.22 \mathrm{GPa}$. This value corresponds very well to the internal stresses of the $270 \mathrm{~nm}$ bottom layer. It seems that the stresses in the SiN and in the first MO-layer are completely relaxed, but still remain in the bottom layer. That corresponds once again very well to the magnetic measurements, because the hysteresis loop from the bottom layer shows still a perfect PMA (Fig. 3b), while the first MO-layer is in-plane after the implantation* (Fig. 3c). Furthermore, the coupling between the first and the bot-

\footnotetext{
*It is not perfect in-plane, because the fluence is just $5 \times 10^{14}$ ions $/ \mathrm{cm}^{2}$.
} 

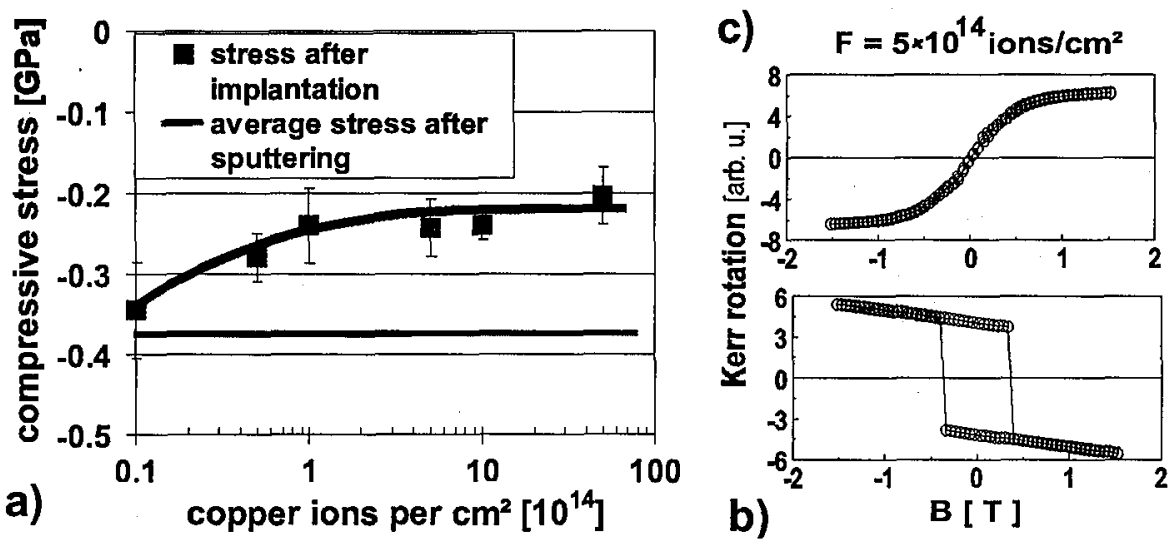

Fig. 3. (a) Change of the internal stresses in dependence on the applied fluence and (b) and (c) the resulting magnetic behaviour of the double layer.

tom MO-layer has disappeared. Because of this we observe a normal Fe-dominated in-plane hysteresis loop.

\section{Summary}

We presented results for two different kinds of ion implanted MO-layers. We found a strong correlation between the magnetical and mechanical behaviour in these layers. They change at a fluence of $1 \times 10^{15}$ ions $/ \mathrm{cm}^{2}$ their PMA to an in-plane anisotropy, while the internal stresses disappear. Between a fluence of $1 \times 10^{13}$ ions $/ \mathrm{cm}^{2}$ and $1 \times 10^{15}$ ions $/ \mathrm{cm}^{2}$ we found transition states with partially PMA and in-plane anisotropy and not completely relaxed states. It is important that the effects do not depend on the kind of ion (TM, RE, non-magnetic), but possible on the energy lost. Furthermore, the ions influence a deeper range than the penetrating depth, but the effect is stopped by a MO-interface.

\section{References}

[1] T. Otto, Ph.D. thesis, Universität Kassel, 1996.

[2] K. Röll, H. Hoffman, Rev. Sci. Instrum. 47, 1183 (1976).

[3] J.F. Ziegler, J.B. Biersack, U. Littmark, The Stopping and Range of Ions in Solids, Pergamon Press, U.K. 1985. 